\title{
Motor expertise affects the unconscious processing of geometric forms
}

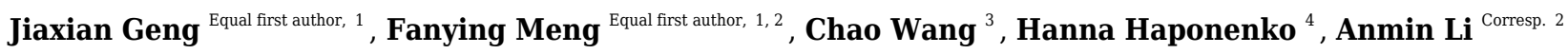 \\ 1 Institute of Physical Education, Huzhou University, Huzhou, Zhejiang, China \\ 2 School of Psychology, Shanghai University of Sport, Shanghai, China \\ 3 Department of Psychology, School of Teacher Education, Huzhou University, Huzhou, Zhejiang, China \\ 4 Department of Psychology, Neuroscience \& Behaviour, McMaster University, Hamilton, Ontario, Canada \\ Corresponding Author: Anmin Li \\ Email address: anminli@sus.edu.cn
}

Background. The unconscious processing of information is an important skill used by competitive athletes to handle the rapidly changing movements of opponents and equipment. Previous studies have shown that unconscious information processing among athletes is better than that among non-athletes in the sports-specific domain. However, it is not yet clear whether athletes also show superior unconscious information processing in the general cognitive domain.

Methods. Twenty-five competitive table tennis players (athletes) and 26 aged-matched non-athletic college students (non-athlete controls) were recruited for this study. Participants first performed a masked priming task that used geometric shapes as primes and targets to examine unconscious information processing in the general cognitive domain. As a control, participants then completed a prime identification task to determine whether they could consciously detect the priming geometric forms. Reaction times and error rates were analyzed to examine whether motor expertise influenced unconscious information processing in the general domain. Nineteen athletes and 17 non-athletes from our present study, which used general stimuli, also participated in our previous study, which used sportspecific stimuli. The strength of the unconscious response priming effect was analyzed to examine whether the effect of motor expertise on unconscious processing could be transferred from a sportsspecific domain to a general domain.

Results. Signal detection analyses indicated that neither athletes nor non-athletes could consciously perceive the priming stimuli. Two-way repeated-measures analyses of variance followed by simple main effects analyses of the masked priming performance, indicating that athletes responded faster and committed fewer errors when the priming stimulus was congruent with the target stimulus. These results suggested that athletes exhibited a significant unconscious response priming effect of geometric forms. By contrast, non-athletes did not respond faster or commit fewer errors for congruent vs. incongruent conditions. No significant difference was detected between athletes and non-athletes in error rates for congruent trials, but athletes committed significantly more errors than non-athletes on incongruent trials. The strength of the unconscious response priming effect that athletes exhibited was greater than that for non-athletes, both in the present study with general stimuli and in our previous study with sport-specific stimuli.

Conclusion. The results indicated that motor expertise facilitated the unconscious processing of geometric forms, suggesting that the influence of motor expertise on unconscious information processing

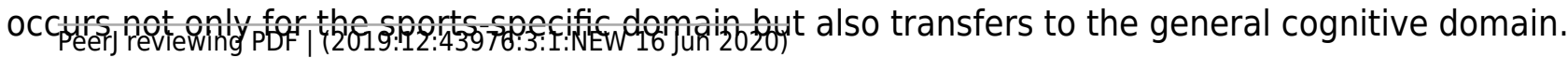


1 Motor expertise affects the unconscious processing of geometric forms

2 Jiaxian Geng ${ }^{1, *}$, Fanying Meng ${ }^{1,2, ~ *}$, Chao Wang ${ }^{3}$, Hanna Haponenko ${ }^{4}$, Anmin $^{2} i^{2}$

$3 \quad{ }^{1}$ Institute of Physical Education, Huzhou University, Huzhou, Zhejiang, China

$4 \quad{ }^{2}$ School of Psychology, Shanghai University of Sport, Shanghai, China

5 '3 Department of Psychology, School of Teacher Education, Huzhou University, Huzhou,

6 Zhejiang, China

$7{ }^{4}$ Department of Psychology, Neuroscience \& Behaviour, McMaster University, Hamilton,

8 Ontario, Canada

$9 \quad$ *These authors contributed equally to this work

11 Corresponding Author:

12 Anmin Li

13 Email address: anminli@sus.edu.cn

15 Abstract

Background. The unconscious processing of information is an important skill used by competitive athletes to handle the rapidly changing movements of opponents and equipment. Previous studies have shown that unconscious information processing among athletes is better than that among non-athletes in the sports-specific domain. However, it is not yet clear whether athletes also show superior unconscious information processing in the general cognitive domain. Methods. Twenty-five competitive table tennis players (athletes) and 26 aged-matched nonathletic college students (non-athlete controls) were recruited for this study. Participants first performed a masked priming task that used geometric shapes as primes and targets to examine unconscious information processing in the general cognitive domain. As a control, participants then completed a prime identification task to determine whether they could consciously detect the priming geometric forms. Reaction times and error rates were analyzed to examine whether motor expertise influenced unconscious information processing in the general domain. Nineteen 
athletes and 17 non-athletes from our present study, which used general stimuli, also participated in our previous study, which used sport-specific stimuli. The strength of the unconscious response priming effect was analyzed to examine whether the effect of motor expertise on unconscious processing could be transferred from a sports-specific domain to a general domain. Results. Signal detection analyses indicated that neither athletes nor non-athletes could consciously perceive the priming stimuli. Two-way repeated-measures analyses of variance followed by simple main effects analyses of the masked priming performance, indicating that athletes responded faster and committed fewer errors when the priming stimulus was congruent with the target stimulus. These results suggested that athletes exhibited a significant unconscious response priming effect of geometric forms. By contrast, non-athletes did not respond faster or commit fewer errors for congruent vs. incongruent conditions. No significant difference was detected between athletes and non-athletes in error rates for congruent trials, but athletes committed significantly more errors than non-athletes on incongruent trials. The strength of the unconscious response priming effect that athletes exhibited was greater than that for non-athletes, both in the present study with general stimuli and in our previous study with sport-specific stimuli.

Conclusion. The results indicated that motor expertise facilitated the unconscious processing of geometric forms, suggesting that the influence of motor expertise on unconscious information processing occurs not only for the sports-specific domain but also transfers to the general cognitive domain.

\section{Introduction}

Previous studies have shown that behavior can be influenced by visual stimuli that are not consciously perceived; individuals can encode information that lies below their threshold for conscious awareness (Ortells, Kiefer, Castillo, Megias, \& Morillas, 2016; Tseng et al., 2016; Zovko \& Kiefer, 2013). In competitive sports, athletes, especially those engaged in sports that require open skills, frequently process movement information unconsciously because of the 
54 limited time available prior to motor reaction (Kibele, 2006). A widely used paradigm to study

unconscious information processing is the masked priming task (Kiefer, 2012; Kiefer \& Martens, 2010). The unconscious response priming effect is considered an indirect measure in evaluating the processing of masked primes (Ansorge, Kunde, \& Kiefer, 2014). The theory of direct parameter specification (DPS, Neumann, 1990; Neumann \& Klotz, 1994) could be used to explain the unconscious response priming effect. According to the DPS theory, once an action plan is established, sensory information can specify response parameters without conscious perception. In the case of the masked priming paradigm, researchers have proved the specification of response parameters by the masked prime through EEG (Leuthold \& Kopp, 1988; Minelli, Marzi, \& Girelli, 2007) and fMRI modeling (Dehanen et.al 1998; Dostilio \& Garraux, 2011; Ulrich \& Kiefer, 2016).

Previous studies have suggested that long periods of practice are required for unconscious or implicit learning (Lewicki, Czyzewska, \& Hoffman, 1987; Wulf \& Schmidt, 1997). With the masked priming paradigm, recent studies found that athletes exhibit stronger unconscious response priming effects than non-athletes in a sport-specific domain, suggesting that athletes show superior unconscious information processing owing to their extensive specialized training (Gueldenpenning, Braun, Machlitt, \& Schack, 2015; Meng, Li, You, \& Xie, 2019). However, whether athletes also show superior unconscious information processing in the general domain is still unknown. Expertise or practice may be an important prerequisite for unconscious information processing (Heinemann, Kiesel, Pohl, \& Kunde, 2010; Kiesel, Kunde, Pohl, Berner, \& Hoffmann, 2009; Reuss, Kiesel, Pohl, \& Kunde, 2015). Athletes are considered experienced individuals because of the extensive motor expertise they have accumulated during their years of training (Guo, Li, \& Yu, 2017; Voss, Kramer, Basak, Prakash, \& Roberts, 2010). Additionally, unconscious information processing is an important skill that enables athletes to deal with rapidly changing movement information (Gueldenpenning, Koester, Kunde, Weigelt, \& Schack, 2011; Kibele, 2006). Hence, researchers are beginning to investigate the relationship between motor expertise and unconscious information processing. For example, using the masked 
81 priming task, Meng et al. (2019) investigated unconscious information processing among table

82

tennis players in the context of objects that represented table tennis - notched circles. The notched circle stimuli were designed based on the characteristic of table tennis and a theoretical model of long-time object memory, called the type-token model. The researchers believed that the circle provided outline information, and the notch orientation provided directional information of the hitting point for the table tennis (Guo et al., 2017; Guo, Li, Lu, \& Gu, 2019).

Results showed that table tennis players, compared to a non-athletic control group, unconsciously perceived visuospatial information and showed a significant unconscious response priming effect. Using a similar methodology, Gueldenpenning et al. (2015) also examined the unconscious processing of complex movements. They researchers found that athletes involved in martial arts training, compared to novices, were able to unconsciously distinguish feint and nonfeint actions and to initiate faster motor responses. Some researchers have suggested that the high perceptual sensitivity and the perceptual-motor common representations among athletes may explain their superiority in unconscious information processing (Gueldenpenning et al., 2011). However, the stimuli that were used in these aforementioned studies are associated with the sports-specific domain. Whether the effect of motor expertise can be transferred from a sports-specific domain to a general domain has not been investigated.

The present study examined whether motor expertise facilitates unconscious information processing in a general masked priming task that uses stimuli unrelated to sports. We recruited a group of competitive table tennis players and a group of aged-matched college students without specific motor expertise to perform a masked priming task and a control prime visibility task. We hypothesized that both athletes and non-athletes would respond faster and commit fewer errors on trials in which prime and target stimuli were congruent than when they were incongruent, displaying a significant unconscious response priming effect. We further hypothesized that the strength of this unconscious response priming effect in athletes would be stronger than that in non-athletes. 
107

108

109

110

111

112

113

114

115

116

117

118

119

120

121

122

123

124

125

126

127

128

129

130

131

\section{Materials and Methods}

\subsection{Participants}

To find an interaction between expertise and response congruency in line with Meng et al. (2019), we calculated the sample size by Gpower3.1 $(\alpha=0.05$, power $=0.80$, effect size $=0.19)$. The result showed that a minimum of 40 participants was required. Twenty-five aged-matched competitive table tennis players ( 9 females; mean age, 20.44 years with a range of 18-23 years) and 26 college students (10 females; mean age, 19.85 years with a range of 18-22 years) were recruited for this study. The competitive table tennis players were considered athletes, and the college students were considered non-athlete controls. All athletes were recruited from Chinese Table Tennis College, Shanghai University of Sport. All of them had obtained the first or second level of the national standard, with a mean table tennis training experience of 7.96 years. The non-athletes were recruited from the School of Economics and Management, Shanghai University of Sport, and had no practical experience with any sports. All participants were right-handed, had normal or corrected-to-normal vision, and had no psychiatric or neurologic illness. All participants provided written informed consent before starting the experiment and received financial compensation after finishing the experiment. This study received approval from the Ethics Committee of Shanghai University of Sport (No. 2018025).

\subsection{Stimuli and equipment}

On the basis of previous studies (Ulrich \& Kiefer, 2016; Zovko \& Kiefer, 2013), we selected images of four black geometric forms (circle, diamond, ellipsoid, and square) as primes and targets. Two images that contained many randomly oriented lines were designed as masks. The stimuli (including masks) were presented on a gray background (RGB values of 128,128, and 128) with a size of $7 \times 7 \mathrm{~cm}$ and subtended a visual angle of $6.69^{\circ}$ (horizontally and vertically) from a viewing distance of $60 \mathrm{~cm}$. 
132

133

134

135

136

137

138

139

140

141

142

143

144

145

146

147

148

149

150

151

152

153

154

155

156

A Dell computer with a 16-inch VGA display (frequency $60 \mathrm{~Hz}$, resolution $1024 \times 768$ ) was used for stimulus presentation. The E-prime 2.0 software package (Psychology Software Tools, Pittsburgh, PA, USA) was used for response sampling.

\subsection{Procedure and tasks}

\subsubsection{Masked priming task}

During the experiment, the experimenter strictly adhered to the rules and maintained a neutral attitude. Participants were instructed to respond to the geometric form of the target stimuli as soon as possible under the premise of ensuring the correct response by pressing the appropriately assigned key on a computer keyboard. An image of a circle or a diamond was assigned to the " $\mathrm{f}$ " key, and an image of an ellipsoid or a square was assigned to the " $\mathrm{j}$ ” key. The reaction was counterbalanced across participants; half the participants followed the aforementioned stimulusresponse key assignment, and the remaining participants followed the opposite stimulus-response key assignment.

The sequence of each trial in the masked priming task is shown in Fig. 1 and occurred as follows: (1) a fixation cross appeared for $750 \mathrm{~ms}$; (2) a forward mask (i.e., an image containing many randomly oriented lines) appeared for $200 \mathrm{~ms}$; (3) the priming stimulus appeared for $33 \mathrm{~ms}$; (4) a backward mask (i.e., another image containing many randomly oriented lines that differed from the forward mask) appeared for $33 \mathrm{~ms}$; (5) the target stimulus appeared for $500 \mathrm{~ms}$; (6) a blank screen appeared for $1000 \mathrm{~ms}$. Participants were required to report their decision (i.e., press the appropriate key) within $1500 \mathrm{~ms}$. The inter-trial interval varied randomly from 1000 to 1500 ms.

\section{(Insert Figure 1 about here)}

All participants were given 24 practice trials before the formal experiment began. The masked priming task followed and included four blocks of 168 total trials. The relationship between the prime and target allowed for two experimental conditions: congruent, for which the prime and 
157

158

159

160

161

162

163

164

165

166

167

168

169

170

171

172

173

174

175

176

177

178

179

180 target included geometric forms belonging to the same response category, and incongruent, for which the prime and target included geometric forms belonging to the different response category. Within each block, half the trials were congruent, and half were incongruent. The four geometric forms appeared equally often as primes and targets and were varied across trials to avoid the repetition response priming effect.

\subsubsection{Prime identification control task}

To assess whether or not participants consciously perceived the prime, they participated in a task that provided a subjective measure of conscious awareness after finishing the masked priming task. This measure was conducted in an interview. The sequence and content of questions occurred as follows: (1) In addition to the fixation, two random line patterns, and target graphics, what else did you see? (2) Did you see any flashing stimulus between the two random line patterns? (3) What do you think this flashing stimulus was? (4) Did you see a geometric form during this flashing stimulus? (5) What specific geometric form did you see? In addition, the participants performed an objective measure of prime identification. Apart from 24 practice trials, the prime identification task included 64 trials (32 congruent trials and 32 incongruent trials). Participants were informed of the existence of the prime. The prime identification task procedure was identical to the masked priming task in that participants were asked to respond to the geometric form of the primes with the same stimulus-response key assignment as was used for the priming task. There was no time pressure for participants in the prime identification task. Participants were required to make their best guess when they were unable to determine the form of the primes.

\subsection{Statistical analysis}

\subsubsection{Identification rates}

We used the statistic $d^{\prime}$ as a signal detection measure to assess prime visibility. Consistent with

Peer] reviewing PDF | (2019:12:43976:3:1:NEW 16 Jun 2020) 
181 the work of Green and Swets (1966), the present study considered a correct response to the target

182

183

184

185

186

187

188

189

190

191

192

193

194

195

196

197

198

199

200

201

202

203

204

205

stimulus on a congruent trial a hit and an erroneous response to the target stimulus on an

incongruent trial a false alarm (Kiefer \& Martens, 2010). The hit rates and false-alarm rates were

calculated for each participant, and the identification rate $d^{\prime}$ was calculated from each

participant's hit and false-alarm rates.

To obtain an objective measure of the ability to identify the geometric prime form presented

between the two masks, one-sample $t$-tests were performed to determine whether the

identification performances of athletes and non-athletes were distributed around the level of

chance, at $50 \%$ (which is indicated by $d^{\prime}=0$ ). We then compared the identification rates of

priming stimuli $\left(d^{\prime}\right)$ between athletes and non-athletes through independent $t$-test. The Bayes

Factor was employed to evaluate the null-hypothesis (a Cauchy distribution (scale $=0.707$ ) as

the prior for the one-sample $t$-test). To rule out that any unconscious response priming effect

observed among athletes correlated with prime visibility, Pearson's correlation between the

identification rate $d^{\prime}$ and the size of the unconscious response priming effect was performed for the group of athletes.

\subsubsection{Reaction times and response errors}

To minimize errors caused by incorrect and extreme values, incorrect and missed trials $(17.52 \%)$ and reaction times (RTs) that deviated by more or less than three standard deviations $(0.83 \%)$ were removed from further analysis. The mean RT of the correct responses and the mean error rates (ERs) were calculated for each participant and each experimental condition. Two-way repeated-measures analyses of variance (ANOVAs) were used to examine the mean RTs and ERs, with the within-subjects factor being response congruency (congruent vs. incongruent) and the between-subjects factor being expertise (athletes vs. non-athletes).

\subsubsection{The strength of the unconscious response priming effect}

To better illustrate whether the effect of motor expertise on unconscious processing could be 
206

207

208

209

210

211

212

213

214

215

216

217

218

219

220

221

222

223

224

225

226

227

228

229

230

transferred from a sports-specific domain to a general domain, we first selected the 19 athletes (6 females; mean age, 20.63 years with a range of 18-23 years) and 17 non-athletes ( 5 females; mean age, 20.06 years with a range of 18-22 years), these subjects participated in the present study with general stimuli, as well as the previous study with sport-specific stimuli. The strength of the unconscious response priming effect (the reaction time on incongruent trials minus the reaction time on congruent trials) was calculated for each participant and each experimental condition. Two-way repeated-measures analyses of variance (ANOVAs) were used to examine the strength of the unconscious response priming effect, with the within-subject factor being stimulation type (general stimuli vs. sport-specific stimuli) and the between-subjects factor being expertise (athletes vs. non-athletes).

\section{Results}

\subsection{Prime visibility}

During the subjective measurement, 10 athletes and 7 non-athletes reported that they saw some images between the random line patterns, but the exact content of the images was unknown. Additionally, four athletes and two non-athletes reported that they saw some regular forms, but it was not clear what the forms were. The other participants did not perceive any images except the fixation, random lines patterns, and target graphics.

The signal detection analysis on the performance of participants to identify the masked primes indicated that, for athletes, $d^{\prime}$ was 0.14 , which did not deviate significantly from zero $\left(t_{(24)}=0.97\right.$, $p=0.341$ ). In addition, for non-athletes, $d^{\prime}$ was 0.06 , which also did not differ significantly from zero $\left(t_{(25)}=0.81, p=0.425\right)$. Furthermore, no significant difference was found between the $d^{\prime}$ values of athletes and non-athletes $\left(t_{(49)}=0.48, p=0.636\right)$. These results indicated that neither athletes nor non-athletes could consciously perceive the masked primes.

The distribution of the identification rates $d^{\prime}$ (Kolmogorov-Smirnov $\left.=0.14, p=0.20\right)$ and the size of the unconscious response priming effect (Kolmogorov-Smirnov $=0.10, p=0.20$ ) for 
231 athletes were normal. For athletes, the identification rates $d^{\prime}$ and the size of the unconscious

232 response priming effect did not correlate with each other $(\mathrm{r}(25)=-0.19, p=0.377)$, suggesting

233 that the unconscious response priming effect of the athletes was not the result of their awareness 234 of the masked primes.

235

236

237

238

239

240

241

242

243

244

245

246

247

248

249

250

251

252

253

254

\subsection{Reaction times and response errors}

The results of a two-way repeated-measures ANOVA examining RTs revealed a significant main effect of response congruency $\left(F_{(1,49)}=11.44 ; p=0.001 ; \eta_{p}^{2}=0.19\right)$. The interaction between expertise and response congruency was also significant $\left(F_{(1,49)}=8.43 ; p=0.006 ; \eta_{p}^{2}=\right.$ 0.15). A simple effects analysis of the interaction showed that athletes responded faster on congruent trials than on incongruent trials (mean \pm standard error: congruent, $489.15 \pm 9.75 \mathrm{~ms}$; incongruent, $500.33 \pm 8.99 \mathrm{~ms} ; p=0.00)$. By contrast, there was no significant difference in reaction times between congruent and incongruent trials for non-athletes (congruent, $500.56 \pm$ $9.56 \mathrm{~ms}$; incongruent, $501.41 \pm 8.82 \mathrm{~ms} ; p=0.734)$. In addition, the main effect of expertise did not reach statistical significance $\left(F_{(1,49)}=0.23 ; p=0.633 ; \eta_{p}^{2}=0.01\right)$ (Fig. 2).

(Insert Figure 2 about here)

An analogous two-way repeated-measures ANOVA on ERs revealed a significant main effect of response congruency $\left(F_{(1,49)}=38.39 ; p=0.00 ; \eta_{p}^{2}=0.44\right)$. The interaction between expertise and response congruency was also significant $\left(F_{(1,49)}=22.80 ; p=0.00 ; \eta_{p}^{2}=0.32\right)$. For response congruency, the analysis of the simple effects of the interaction showed that athletes committed fewer errors on congruent trials than on incongruent trials (congruent, $13.00 \% \pm 1.59 \%$; incongruent, $26.36 \% \pm 2.08 \% ; p=0.001)$. By contrast, there was no significance difference in ERs between congruent and incongruent trials for non-athletes (congruent, $14.27 \% \pm 1.56 \%$; incongruent, $16.00 \% \pm 2.04 \% ; p=0.572$ ). For expertise, athletes committed more errors than non-athletes on incongruent trials (athletes, $26.36 \% \pm 2.08 \%$; non-athletes, $16.00 \% \pm 2.04 \% ; p=$ 
2550.00 ), but not on congruent trials (athletes, $13.00 \% \pm 1.59 \%$; non-athletes, $14.27 \% \pm 1.56 \% \% ; p$

$256=0.315)$. However, the main effect of expertise did not reach significance $\left(F_{(1,49)}=3.94 ; p=\right.$

$\left.257 \quad 0.053 ; \eta_{p}^{2}=0.07\right)($ Fig. 3).

258 (Insert Figure 3 about here)

259

260

261

262

263

264

265

266

267

268

269

270

271

272

273

274

275

276

277

\subsection{The strength of the unconscious response priming effect}

The results of a two-way repeated-measures ANOVA examining the strength of the unconscious response priming effect revealed a significant main effect of expertise $\left(F_{(1,34)}=\right.$ $\left.12.58 ; p=0.001 ; \eta_{p}^{2}=0.27\right)$. The strength of the unconscious response priming effect that athletes showed was higher than for non-athletes (athletes, $15.13 \pm 2.65 \mathrm{~ms}$; non-athletes, 1.46 $\pm 2.80 \mathrm{~ms})$. The main effect of stimulation type did not reach statistical significance $\left(F_{(1,34)}=\right.$ $\left.0.05 ; p=0.818 ; \eta_{p}^{2}=0.00\right)$. The interaction between expertise and stimulation type did not reach statistical significance $\left(F_{(1,34)}=1.51 ; p=0.228 ; \eta_{p}^{2}=0.04\right)($ Fig. 4$)$.

(Insert Figure 4 about here)

\section{Discussion}

Although unconscious information processing among athletes is reportedly better than that of non-athletes in the sports-specific domain, previous research has not fully shown whether athletes also show superior unconscious information processing in the general domain. Thus, to determine whether motor expertise is associated with unconscious information processing in the general domain, our experiment compared the performance between athletes and non-athletes on a masked priming task that used geometric forms unrelated to the sport domain as the prime and target. The athletes belonged to a group of competitive table tennis players who had extensive motor expertise, and the non-athletes belonged to a group of college students without specific motor expertise. Our primary results showed that athletes responded faster and committed fewer 
278

279

280

281

282

283

284

285

286

287

288

289

290

291

292

293

294

295

296

297

298

299

300

301

302

303

304

errors on trials in which the prime and target stimuli were congruent (i.e., required the same response) than on trials in which they were incongruent. By contrast, there was no significant difference in reaction times or error rates between congruent and incongruent trials for nonathletes. Moreover, athletes showed a significant unconscious response priming effect both in the masked priming task with general stimuli and the masked priming task with sport-specific stimuli.

Athletes showed a significant unconscious response priming effect compared to non-athletes, which suggests that athletes exhibited superior unconscious information processing. This finding is consistent with the results of a previous study showing that motor expertise promotes unconscious response inhibition in the general cognitive domain (You et al., 2018). We speculate that motor expertise possessed by athletes facilitates unconscious information processing. A characteristic of competitive sports is that they require rapid and changeable responses. Given the Olympic motto "faster, higher, and stronger" (Coubertin, 2008), competitive sports, especially open skills sports, have developed so that the movement speed of athletes and sports equipment exceeds the perception threshold of individuals most of the time. For example, in table tennis, the ball speeds may be up to $144-180 \mathrm{~km} / \mathrm{h}$, and the contact time between the ball and the racket is only approximately $0.01 \mathrm{~s}$ (Bootsma \& Van Wieringen, 1990). With such time pressure, the decisions made by competitive athletes are usually without any explicit evaluation of the perceived information (Raab \& Johnson, 2008; Williams \& Ward, 2007). Table tennis is an open skills sport in which athletes are required to react in a dynamically changing environment, and likely because of their years of training, these athletes show more unconscious behaviors compared with non-athletes (Wang et al., 2013; Yu, Chan, Chau, \& Fu, 2017). We further found that the strength of the unconscious response priming effect among athletes was not affected by stimulation type, suggesting that the influence of motor expertise on unconscious processing can be transferred from a sports-specific domain to a general domain. The masked priming paradigm used in the present study closely replicated the time pressure of an open skills sport and was favorable for the performance of the athletes. 
305

306

307

308

309

310

311

312

313

314

315

316

317

318

319

320

321

322

323

324

325

326

327

328

329

330

331

Schütz-Bosbach and Prinz (2007) have suggested that perceptual sensitivity is a prerequisite for unconscious processing. We selected geometric forms as prime and target stimuli because they are considered well-known to most people (Kiesel et al., 2009). Thus, we believed that both athletes and non-athletes would show high perceptual sensitivity to these geometric forms. However, our results showed that only athletes exhibited a significant unconscious response priming effect whereas the non-athletes did not. This result was in line with Guo et al. (2017), which found that regardless of priming with sport-specific stimuli or general stimuli, table tennis players had better performance in perceptual observation and motion control compared to nonathletes. According to the results of the present study and previous research, we speculated that athletes had higher perceptual sensitivity to geometric forms in comparison with non-athletes. To accurately judge the flight trajectory of the ball, as well as its spinning and land point, table tennis players may rely heavily on the visual imagery system. Athletes may process movement information through image coding during daily training and competition. Therefore, it may be the case that athletes with extensive special training showed higher perceptual sensitivity to the sport-specific stimuli and general geometric forms compared to non-athletes.

In fast-ball sports, the perception-action coupling capacities may play a critical role in motor expertise (Ranganathan, \& Carlton, 2007). These capacities are not limited to sport-specific scenarios, but may also differentiate athletes and non-athletes in general cognitive tasks (Mallek, Benguigui, Dicks, \& Thouvarecq, 2017). Compared to the number of words used as prime and target stimuli in previous studies (Martens, Ansorge, \& Kiefer, 2011; Ulrich, Adams, \& Kiefer, 2014), the number of geometric forms used in the present study was small, and each geometric shape had a unique physical feature. Participants may have stored the geometric forms and their corresponding reactions in their working memory, subsequently forming a stimulus-reaction coupling (Kiesel, Kunde, Poh, \& Hoffmann, 2006; Wang, Huo, \& Wang, 2012). The present results indicated that the performance of athletes was significantly influenced by the presentation of the geometric forms despite perception being unconscious, whereas the performance of nonathletes was not affected by the presence of geometric forms whatsoever. Athletes established an 
332

333

334

335

336

337

338

339

340

341

342

343

344

345

346

347

348

349

350

351

352

353

354

355

356

357

358

unconscious association between the stimuli and motor responses, whereas the non-athletes did not. The motor reactions of the athletes were likely facilitated or disrupted by the unconsciously perceived primes, with the unconsciously perceived geometric shapes activating the previously established stimulus-response assignment and triggering a motor response for situations of prime-target congruency. The response to the target would have been facilitated as a result of being pre-activated by the prime. By contrast, when the prime and target stimuli required a different response (i.e., were incongruent), the response to the target would have been interfered because the pre-activated response was inconsistent with the required target response. Athletes would have needed to timely inhibit the incorrect motor response tendency during incongruent trial types. Hence, athletes responded faster and committed fewer errors on congruent trials than on incongruent trials. In the present study, athletes seemed to learn the rules faster and more effectively than non-athletes, permitting the establishment of a more stable unconscious connection between the stimuli and motor response. By contrast, this connection was not stable in non-athletes and did not reach an automatic level. Thus, the stimuli presented below the threshold of consciousness didn't influence the performance of non-athletes. Although there was no unconscious response priming effect for non-athletes, error rates showed that non-athletes committed more errors on incongruent trials than on congruent trials, which paralleled a similar result with athletes. An aposteriori sample size based on the effect sizes of error rates was calculated in Gpower3.1, with the result showing that a minimum of 80 participants was needed with $\alpha=0.05$, power $=0.80$, effect size $=0.32$. In order to find an equivalent main effect of response congruency, more non-athletes would be needed in future studies. We also found that the error rates among athletes were higher than those among non-athletes on incongruent trials, suggesting that stimulus-response conflict that was unconsciously perceived was stronger than non-athletes.

Moreover, our results showed that non-athletes did not exhibit a significant unconscious response priming effect. However, this finding was inconsistent with previous studies (Klotz \& Neumann, 1999; Neumann \& Klotz, 1994; Ulrich \& Kiefer, 2016). For instance, the study of 
359 Klotz and Neumann (1999), which tested geometric stimuli on normal subjects, found that the 360 participants exhibited significant unconscious response priming effect, suggesting that motor 361 activation could be trigged without conscious discrimination. We speculated that the reason for 362 the inconsistent results between us and Klotz and Neumann (1999) might be the level of task

363

364

365

366

367

368

369

370

371

372

373

374

375

376

377

378

379

380

381

382

383

384

385 difficulty. Only two geometric forms were used in the study of Klotz and Neumann (1999), resulting in fewer the possible prime-target combinations compared to our current study.

Participants in their study made a response according to the spatial position of the target (e.g., if the target on the right, participants made a response by pressing the right button). Thus, we believed that the localization task in the present study was more difficult than the study of Klotz and Neumann (1999). Kibele $(2001,2006)$ suggested that unconscious information processing may be obstructed in complex tasks. Along a similar vein, in studying the neural signature of the unconscious response priming effect, Ulrich and Kiefer (2016) recruited college students to participant in a masked priming task that also used geometric forms. They found that the difference in RTs between congruent and incongruent trials was significant, with the RTs for congruent trials averaging $575 \mathrm{~ms}$ and the RTs for incongruent trials averaging $588 \mathrm{~ms}$. In contrast, the difference was not significant in ER because of slow decisions. In the present study, the RT range is $500-501 \mathrm{~ms}$, indicating that non-athletes focused on the current task with their rapid responses not compromising accuracy. So, it is possible that non-athletes would have been able to show an unconscious response priming effect provided they made slower responses. In turn, perhaps this would produce results, comparable to Ulrich and Kiefer (2016). Although participants were normal subjects in both studies, the reaction strategies of these two groups might be different. Based on the previous researches (Braver, Cole, \& Yarkoni, 2010; Capa \& Bouquet, 2018; Van Gaal, Scholte, Lamme, Fahrenfort, \& Ridderinkhof, 2011), we speculated that the group difference might be responsible for the inconsistent results. Future studies could systematically explore the effect of individual differences or group differences on unconscious information processing both in the general and sport-specific domain.

Our findings, together with the results from previous studies, not only add further evidence to 
386

387

388

389

390

391

support that expertise is an important determinant of unconscious processing but also provide preliminary evidence that, compared with non-athletes, athletes exhibit superior unconscious information processing in the general domain. Thus, the influence of motor expertise on unconscious information processing may be transferable from the sports-specific domain to the general cognitive domain.

\section{Limitations}

The present study had some limitations. Firstly, participants may have judged it too difficult to use full cognitive effort in consciously perceiving the prime. Thus, subjective threshold measurements such as the 5-point Likert scale should be considered in future studies, as it would contribute to a more enhanced data precision in comparison to the binary interview procedure in the prime identification task. Secondly, the athletes played table tennis, which is an open skills sport. In order to better identify the facilitation of motor expertise on unconscious information processing in the general cognitive domain, athletes who play closed skills sports should also be recruited in future studies. Thirdly, although the present study described the influence of motor expertise on the unconscious executive control of the general domain on a behavior level, the neural signature of this phenomenon remains unclear and warrants future exploration.

\section{Conclusions}

The findings of our study provide evidence that motor expertise facilitates the unconscious processing of geometric forms, indicating that the influence of motor expertise on unconscious executive control is not just sports-specific but can be transferred to the general cognitive domain.

\section{References}

Ansorge, U., Kunde, W., \& Kiefer, M. (2014). Unconscious vision and executive control: how unconscious processing and conscious action control interact. Consciousness \& Cognition, 27(27), 268-287. doi: 10.1016/j.concog.2014.05.009 
411 Bootsma, R. J., \& Van Wieringen, P. C. (1990). Timing an attacking forehand drive in table

412

413

414

415

416

417

418

419

420

421

422

423

424

425

426

427

428

429

430

431

432

433

434

435

436

437 tennis. Journal of Experimental Psychology, 16(1), 21-29.

Braver, T. S., Cole, M., \& Yarkoni, T. (2010). Vive les differences! Individual variation in neural mechanisms of executive control. Current Opinion in Neurobiology, 20(2), 242250. doi: 10.1016/j.conb.2010.03.002

Capa, R. L., \& Bouquet, C. A. (2018). Individual differences in reward sensitivity modulate the distinctive effects of conscious and unconscious rewards on executive performance. Frontiers in Psychology, 9, 148. doi: 10.3389/fpsyg.2018.00148

Coubertin, P. (2008). Olympic Manifesto. Beijing: People's Publishing House.

Dehaene, S., Naccache, L., Clech, G. L., Koechlin, E., Mueller, M., Dehaene-lambertz, G., ... \& Bihan, D. L. (1998). Imaging unconscious semantic priming. Nature, 395(6702), 597-600. doi: $10.1038 / 26967$

Dostilio, K., \& Garraux, G. (2011). Automatic stimulus-induced medial premotor cortex activation without perception or action..PLOS ONE, 6(2), e16613. doi: 10.1371/journal.pone.0016613

Green, D. M., \& Swets, J. A. (1966). Signal detection theory and psychophysics. NY: Wiley.

Gueldenpenning, I., Braun, J. F., Machlitt, D., \& Schack, T. (2015). Masked priming of complex movements: perceptual and motor processes in unconscious action perception. Psychological Research-Psychologische Forschung, 79(5), 801-812. doi: $10.1007 / \mathrm{s} 00426-014-0607-\mathrm{z}$

Gueldenpenning, I., Koester, D., Kunde, W., Weigelt, M., \& Schack, T. (2011). Motor expertise modulates the unconscious processing of human body postures. Experimental Brain Research, 213(4), 383-391. doi: 10.1007/s00221-011-2788-7

Guo, Z., Li, A., Lu, Q., \& Gu, N. (2017). Neural efficiency of table tennis players during image recognition: an EEG study. Journal of Capital University of Physical Education and Sports, 29(1), 78-83. doi: 10.14036/j.cnki.cn11-4513.2017.01.018

Guo, Z., Li, A., \& Yu, L. (2017). "Neural efficiency" of athletes' brain during visuo-spatial task: 
438

439

440

441

442

443

444

445

446

447

448

449

450

451

452

453

454

455

456

457

458

459

460

461

462

463

464

an fMRI study on table tennis players. Frontiers in Behavioral Neuroscience, 11. doi: 10.3389/fnbeh.2017.00072

Heinemann, A., Kiesel, A., Pohl, C., \& Kunde, W. (2010). Masked response priming in expert typists. Consciousness and Cognition, 19(1), 399-407. doi: 10.1016/j.concog.2009.09.003

Klotz, W., \& Neumann, O. (1999). Motor activation without conscious discrimination in metacontrast masking. Journal of Experimental Psychology: Human Perception and Performance, 25(4), 976-992. doi: 10.1037/0096-1523.25.4.97

Kibele, A. (2001). Unbewusste informationsverarbeitung — ein thema für die sportwissenschaft?! [Non-conscious information processing —an issue for sports science?!]. Frankfurt: Peter Lang.

Kibele, A. (2006). Non-consciously controlled decision making for fast motor reactions in sports - a priming approach for motor responses to non-consciously perceived movement features. Psychology of Sport \& Exercise, 7(6), 591-610. doi: 10.1016/j.psychsport.2006.05.001

Kiefer, M. (2012). Executive control over unconscious cognition: attentional sensitization of unconscious information processing. Frontiers in Human Neuroscience, 6. doi: 10.3389/fnhum.2012.00061

Kiefer, M., \& Martens, U. (2010). Attentional sensitization of unconscious cognition: task sets modulate subsequent masked semantic priming. J Exp Psychol Gen, 139(3), 464-489. doi: $10.1037 / \mathrm{a} 0019561$

Kiesel, A., Kunde, W., Poh, C., \& Hoffmann, J. (2006). Priming from novel masked stimuli depends on target set size. Advances in Cognitive Psychology, 2(1), 87-97. doi: 10.2478/v10053-008-0043-y

Kiesel, A., Kunde, W., Pohl, C., Berner, M. P., \& Hoffmann, J. (2009). Playing chess unconsciously. Journal of Experimental Psychology: Learning, Memory and Cognition, 35(1), 292-298. doi: 10.1037/a0014499

Leuthold, H., \& Kopp, B. (1998). Mechanisms of priming by masked stimuli: Inferences From 
465

466

467

468

469

470

471

472

473

474

475

476

477

478

479

480

481

482

483

484

485

486

487

488

489

490

491

event-related brain potentials. Psychological Science, 9(4), 263-269. doi: 10.1111/14679280.00053

Lewicki, P., Czyzewska, M., \& Hoffman, H. G. (1987). Unconscious acquisition of complex procedural knowledge. Journal of Experimental Psychology: Learning, Memory and Cognition, 13(4), 523-530. doi: 10.1037/0278-7393.13.4.523

Mallek, M., Benguigui, N., Dicks, M., \& Thouvarecq, R. (2017). Sport expertise in perceptionaction coupling revealed in a visuomotor tracking task. European Journal of Sport Science, 17(10), 1270-1278. doi:10.1080/17461391.2017.1375014

Martens, U., Ansorge, U., \& Kiefer, M. (2011). Controlling the unconscious: attentional task sets modulate subliminal semantic and visuomotor processes differentially. Psychological Science, 22(2), 282. doi: 10.1177/0956797610397056

Meng, F., Li, A., You, Y., \& Xie, C. (2019). Motor expertise modulates unconscious rather than conscious executive control. Peerj, 7. doi: 10.7717/peerj.6387

Minelli, A., Marzi, C. A., \& Girelli, M. (2007). Lateralized readiness potential elicited by undetected visual stimuli. Experimental Brain Research, 179(4), 683-690. doi: $10.1007 / \mathrm{s} 00221-006-0825-8$

Neumann, O. (1990). Direct parameter specification and the concept of perception. Psychological Research, 52, 207-215. doi: 10.1007/BF00877529

Neumann, O., \& Klotz, W. (1994). Motor responses to nonreportable, masked stimuli: Where is the limit of direct parameter specification? In: C. Umilta, \& M. Moscovitch (Eds.): Attention and Performance 15: Conscious and nonconscious inform a-tion processing. MIT Press: Cambridge MA, 123-150.

Ortells, J. J., Kiefer, M., Castillo, A., Megias, M., \& Morillas, A. (2016). The semantic origin of unconscious priming: behavioral and event-related potential evidence during category congruency priming from strongly and weakly related masked words. Cognition, 146, 143-157. doi: 10.1016/j.cognition.2015.09.012

Raab, M., \& Johnson, J. G. (2008). Implicit learning as a means to intuitive decision making in 
492

493

494

495

496

497

498

499

500

501

502

503

504

505

506

507

508

509

510

511

512

513

514

515

516

517

518 sports. In H. Plessner, C. Betsch, \& T. Betsch (Eds), Intuition in judgement and decision making. New York, London: Taylor \& Francis.

Ranganathan, R., \& Carlton, L. G. (2007). Perception-action coupling and anticipatory performance in baseball batting. Journal of Motor Behavior, 39(5), 369-380. doi:10.3200/JMBR.39.5.369-380

Reuss, H., Kiesel, A., Pohl, C., \& Kunde, W. (2015). Instructed illiteracy reveals expertiseeffects on unconscious processing. Frontiers in Psychology, 6. doi: 10.3389/fpsyg.2015.00239

Schütz-Bosbach, S., \& Prinz, W. (2007). Perceptual resonance: action-induced modulation of perception. Trends in Cognitive Sciences, 11(8), 349-355. doi: 10.1016/j.tics.2007.06.005

Tseng, W.-L., Thomas, L. A., Harkins, E., Pine, D. S., Leibenluft, E., \& Brotman, M. A. (2016). Neural correlates of masked and unmasked face emotion processing in youth with severe mood dysregulation. Social Cognitive and Affective Neuroscience, 11(1), 78-88. doi: $10.1093 / \mathrm{scan} / \mathrm{nsv} 087$

Ulrich, M., Adams, S. C., \& Kiefer, M. (2014). Flexible establishment of functional brain networks supports attentional modulation of unconscious cognition. Human Brain Mapping, 35(11), 5500. doi: 10.1002/hbm.22566

Ulrich, M., \& Kiefer, M. (2016). The neural signature of subliminal visuomotor priming: brain activity and functional connectivity profiles. Cerebral Cortex, 26(6), 2471-2482. doi: 10.1093/cercor/bhv070

Van Gaal, S., Scholte, H. S., Lamme, V. A., Fahrenfort, J. J., \& Ridderinkhof, K. R. (2011). Presma gray-matter density predicts individual differences in action selection in the face of conscious and unconscious response conflict. Journal of Cognitive Neuroscience, 23(2), 382-390. doi: 10.1162/jocn.2010.21444

Voss, M. W., Kramer, A. F., Basak, C., Prakash, R. S., \& Roberts, B. W. (2010). Are expert athletes 'expert' in the cognitive laboratory? a meta-analytic review of cognition and sport expertise. Applied Cognitive Psychology, 24(6), 812-826. doi: 10.1002/acp.1588

Peer) reviewing PDF | (2019:12:43976:3:1:NEW 16 Jun 2020) 
519 Wang, C. H., Chang, C. C., Liang, Y. M., Shih, C. M., Chiu, W. S., Tseng, P., . . . Juan, C. H. 520 (2013). Open vs. closed skill sports and the modulation of inhibitory control. Plos One, $521 \quad 8(2)$, e55773. doi: 10.1371/journal.pone.0055773

522 Wang, P., Huo, P., \& Wang, L. (2012). Processing Levels and Its Prerequisite During Subliminal 523 524

525 526

527

528

529

530

531

532

533

534

535

536

537

538

539

540 Perception: Evidences Based on Visual Masking Priming. Acta Psychologica Sinica, 44(9), 1138-1148. doi: 10.3724/SP.J.1041.2012.01138

Williams, A. M., \& Ward, P. (2007). Anticipation and decision making: exploring new horizons. In G. Tenebaum \& R. Eklund (Eds.), Handbook of sport paychology. New York, NY: Wiley.

Wulf, G., \& Schmidt, R. A. (1997). Variability of practice and implicit motor learning. Journal of Experimental Psychology: Learning, Memory and Cognition, 23(4), 987-1006. doi: 10.1037/0278-7393.23.4.987

You, Y., Ma, Y., Ji, Z., Meng, F., Li, A., \& Zhang, C. (2018). Unconscious response inhibition differences between table tennis athletes and non-athletes. Peerj, 6(3), e5548. doi: $10.7717 /$ peerj. 5548

Yu, Q. H., Chan, C. C. H., Chau, B., \& Fu, A. S. N. (2017). Motor skill experience modulates executive control for task switching. Acta Psychologica, 180, 88-97. doi: 10.1016/j.actpsy.2017.08.013

Zovko, M., \& Kiefer, M. (2013). Do different perceptual task sets modulate electrophysiological correlates of masked visuomotor priming? Attention to shape and color put to the test. Psychophysiology, 50(2), 149-157. doi: 10.1111/j.1469-8986.2012.01492.x 
Figure 1

The masked priming task

Trial sequence of the masked priming task.

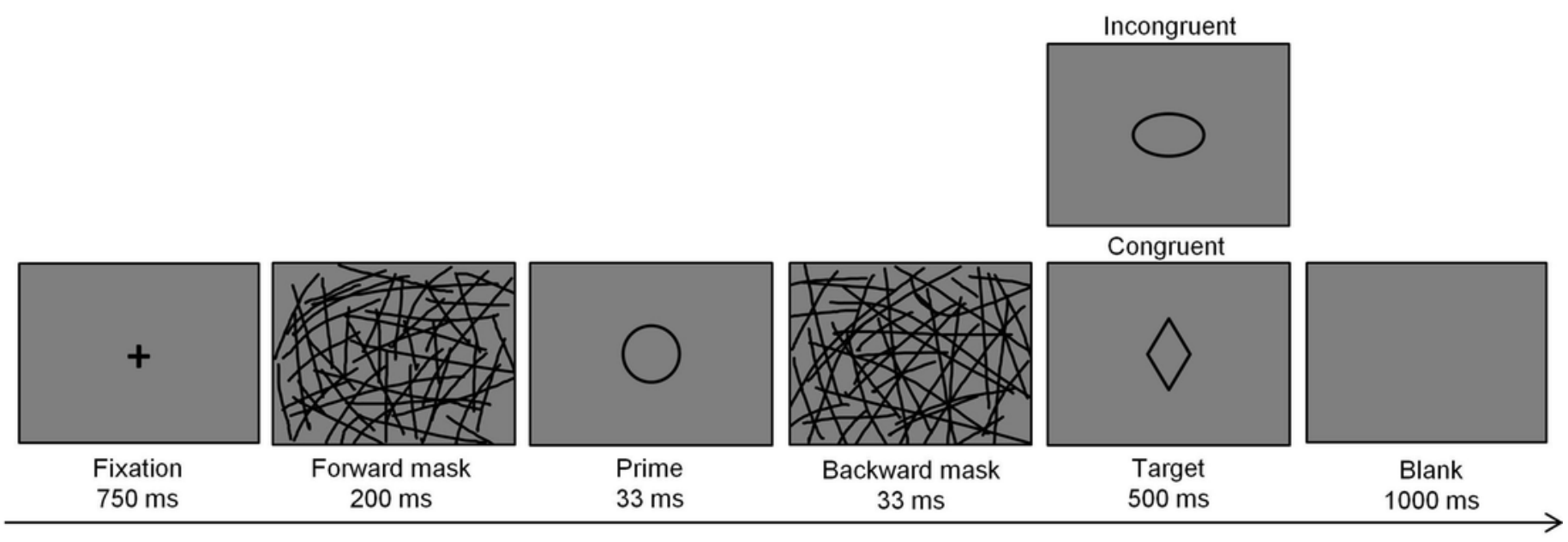




\section{Figure 2}

Mean reaction times for trials with correct responses in the masked priming task for athletes and non-athletes.

The vertical bars represent mean reaction times as a function of response congruency and expertise. White bars represent reaction times of congruent responses, and black bars represent reaction times of incongruent responses. Error bars indicate standard error of mean; *** $p<0.001$ for the indicated comparison. 


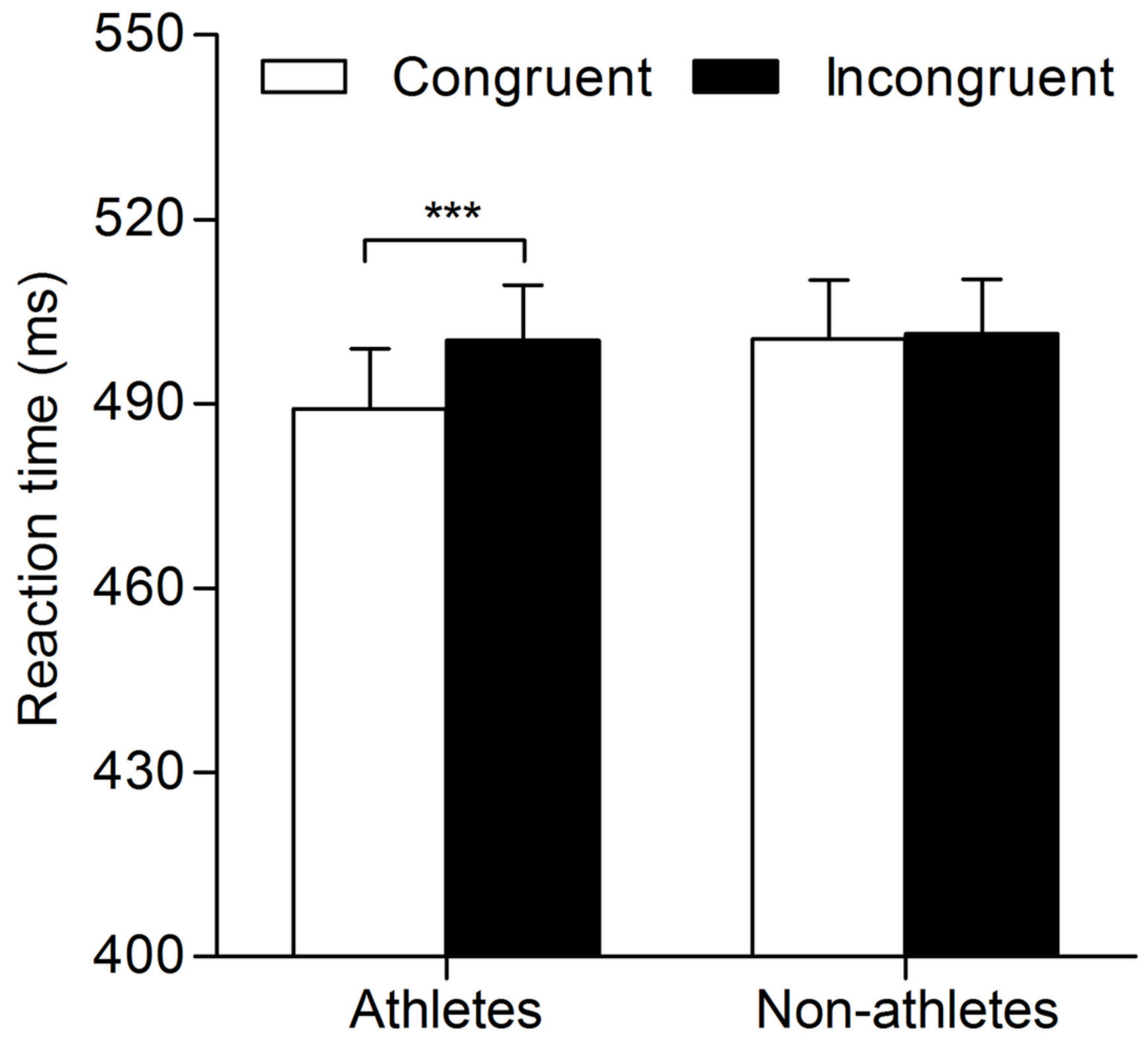




\section{Figure 3}

Mean error rates for athletes and non-athletes in the masked priming task for athletes and non-athletes.

The vertical bars represent mean error rates as a function of response congruency and expertise. White bars represent error rates of congruent responses, and black bars represent error rates of incongruent responses. Error bars indicate standard error of mean; $*^{*} p<0.01$, ${ }^{* * *} p<0.001$ for the indicated comparisons. 


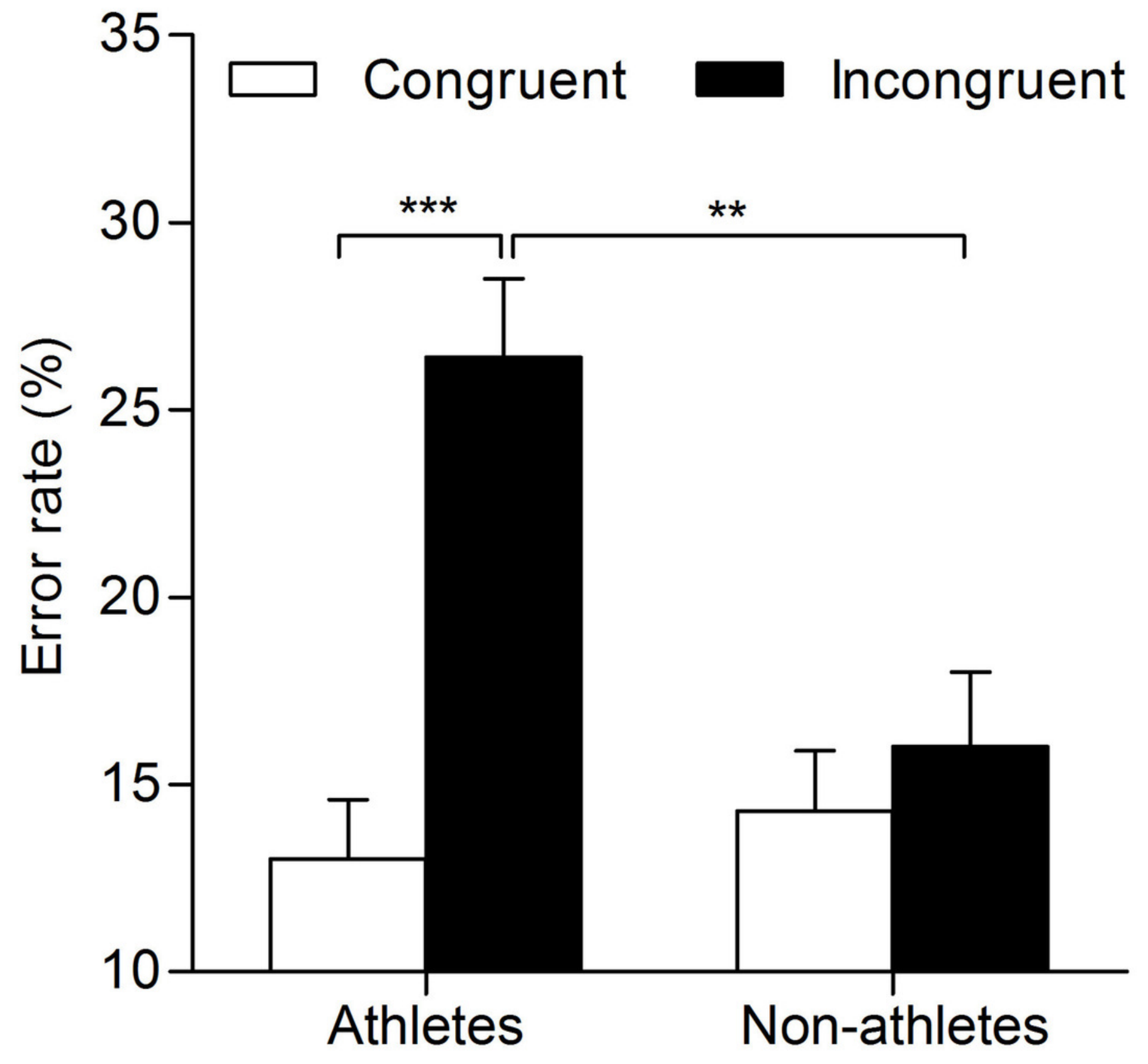


Figure 4

Mean strength of unconscious response priming effect in the masked priming task with general stimuli and sport-specific stimuli for athletes and non-athletes.

The vertical bars represent mean strength of unconscious response priming effect as a function of stimulation type and expertise. White bars represent the strength of unconscious response priming effect of general stimuli, and black bars represent the strength of unconscious response priming effect of sport-specific stimuli.

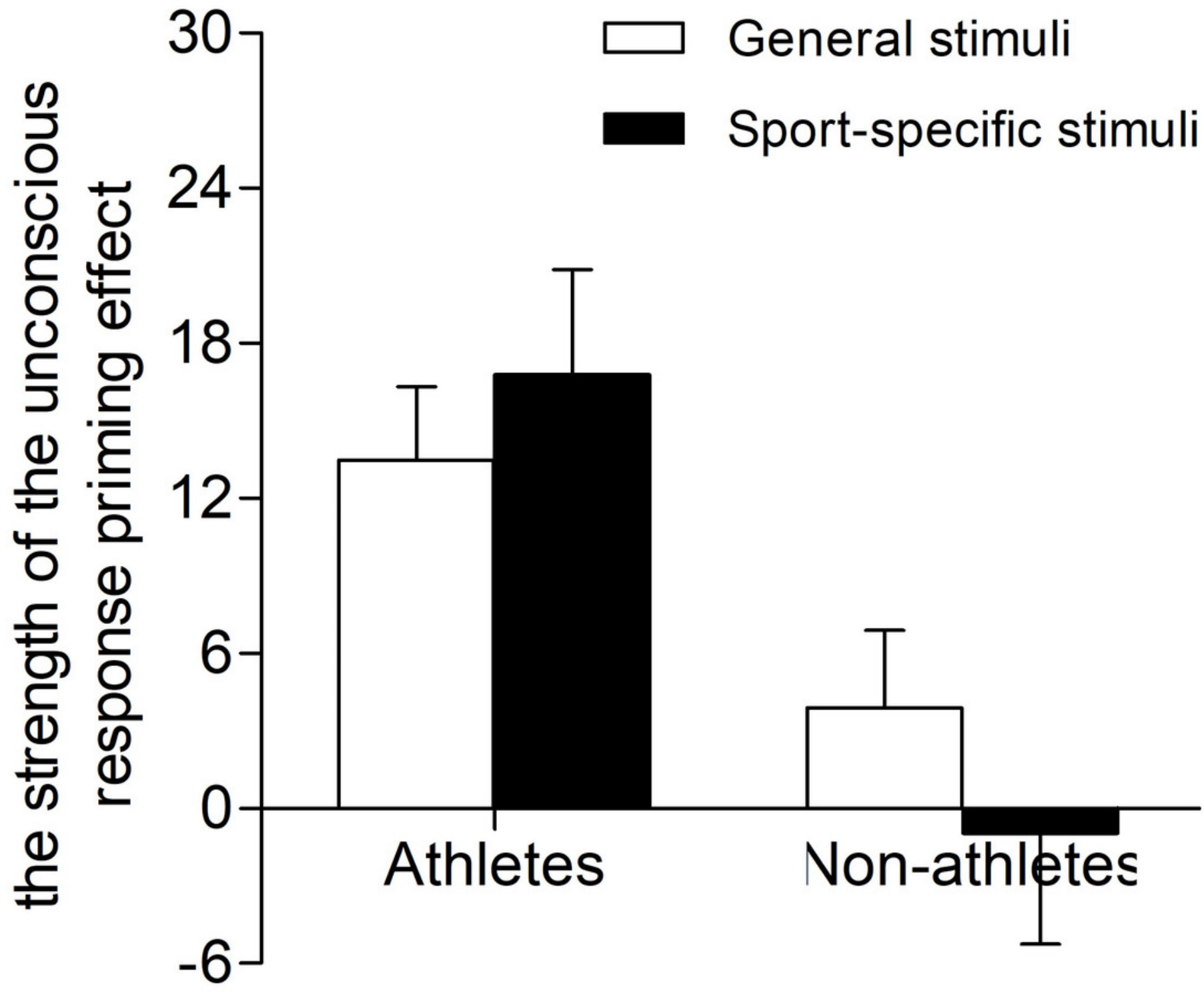

